\title{
UNUSUAL CASE OF A PITUITARY MACROADENOMA INVADING AND ENCASING THE OPTIC TRACK
}

Singapore Med J 2016; 57(7): 410 doi: 10.11622/smedj.2016124

Dear Sir,

We report a rare case of recurrent pituitary macroadenoma encasing and invading the right optic track. While bowing of the optic chiasm is common, invasion and encasement of the optic track has, to the best of our knowledge, never been reported.

A 75-year-old Chinese woman underwent transsphenoidal surgery in June 1999 for pituitary macroadenoma. Postoperative hormonal analysis revealed no anterior pituitary hormonal deficiency. However, the patient had polyuria; desmopressin was started but subsequently
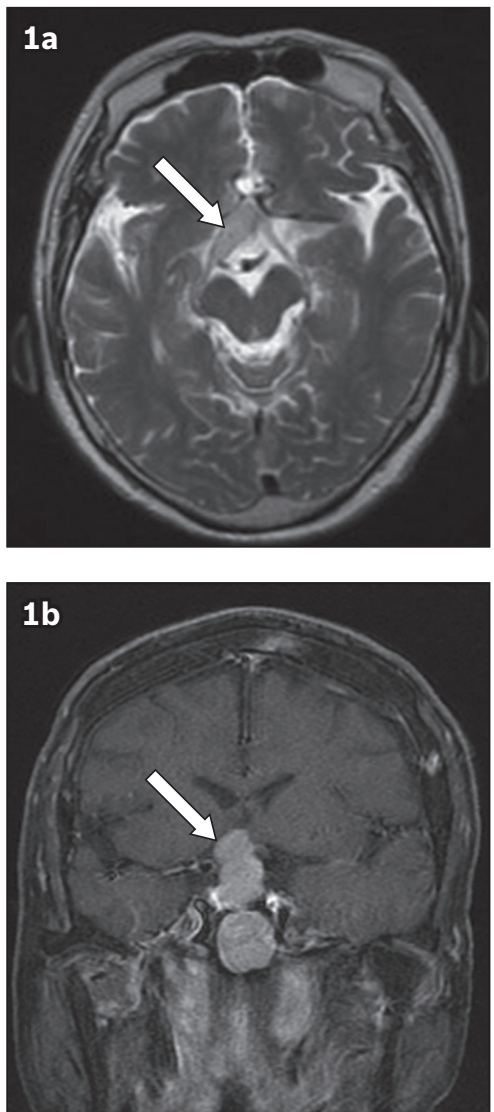

Fig. 1 MR images show (a) pituitary macroadenoma encasing and invading the right optic track (arrows) and (b) extension into the right cavernous sinus.

The transsphenoidal approach is generally favoured over transcranial surgery for excision of most pituitary adenomas, as the former causes less injury to the pituitary gland and allows rapid recovery of pituitary endocrinological function and visual impairment. However, controversy still exists over the surgical approach for adenomas with suprasellar extensions. Regular careful observation is recommended after surgery, with hormonal replacement when needed. Radiotherapy is reserved for controlling a residual tumour that is surgically unresectable, especially when cavernous sinus invasion is present and for the prevention of late recurrences. ${ }^{(5)}$

Yours sincerely,

Nishini Hekha ${ }^{1}$, Yih-Yian $\underline{\text { Sitoh }}^{1}$, Francis Kim-Hoong $\underline{\text { Hui }}^{1}$

${ }^{1}$ Department of Neuroradiology, National Neuroscience Institute, Singapore. nishinihekha@yahoo.com

\section{References}

1. Matsuyama J, Kawase T, Yoshida K, et al. Management of large and giant pituitary adenomas with suprasellar extensions. Asian J Neurosurg 2010; 5:48-53.

2. Hardy J, Vezina JL. Transsphenoidal neurosurgery of intracranial neoplasm. Adv Neurol 1976; 15:261-73.

3. Wilson CB. A decade of pituitary microsurgery. The Herbert Olivecrona lecture. J Neurosurg 1984; 61:814-33.

4. Knosp E, Steiner E, Kitz K, Matula C. Pituitary adenomas with invasion of the cavernous sinus space: a magnetic resonance imaging classification compared with surgical findings. Neurosurgery 1993; 33:610-7.

5. Mortini P, Barzaghi R, Losa M, Boari N, Giovanelli M. Surgical treatment of giant pituitary adenomas: strategies and results in a series of 95 consecutive patients. Neurosurgery 2007; 60:993-1002. 